\title{
G6PD Deficiency Overrepresented Among Pediatric COVID-19 Cases in One Saudi Children Hospital
}

Maryam Al-Aamri ${ }^{\mathrm{a}}$, Fatima Al-Khalifa ${ }^{\mathrm{a}}$, Fawatim Al-Nahwi ${ }^{\mathrm{a}}$, Heba Al-Ameer ${ }^{\mathrm{a}}$, Sameer AlAbdi ${ }^{\mathrm{b}, \mathrm{c}, \mathrm{d}}$

${ }^{a}$ Department of Pediatrics, Maternity and Children Hospital, Al-Ahsa, Ministry of Health, Saudi Arabia.

${ }^{b}$ Department of Pediatrics, King Abdulaziz Hospital, Ministry of the National Guard-Health Affairs, Al-Ahsa, Saudi Arabia.

${ }^{c}$ King Abdullah International Medical Research Center, Al-Ahsa, Saudi Arabia.

${ }^{\mathrm{d}}$ King Saud Bin Abdulaziz University for Health Sciences, Al-Ahsa, Saudi Arabia.

Cross ponding Author: Sameer Al-Abdi (abdis@ ngha.med.sa)

PO Box 2477, Al-Ahsa 31982, Saudi Arabia

Mobile: +966569031455

Abstract

Fluorescent spot test for glucose-6-phosphate dehydrogenase (G6PD) deficiency was performed in 5 boys and 14 girls who had confirmed COVID-19. Out of those, 4 (80\%) boys and $5(36 \%)$ girls were found to be G6PD deficient.

\section{Main Text}

On March 12, 2020, the first case of coronavirus disease 19 (COVID-19) was diagnosed in the Al-Ahsa area, Saudi Arabia [1]. Until the end of April 2020, all children of less than 14 years old with confirmed COVID-19 were admitted to the Maternity and Children hospital in Al-Ahsa area. During this period, 29 children (8 boys and 21girls) were admitted. All these cases were either asymptomatic or with a mild COVID-19. Fluorescent spot test (FST) for glucose-6-phosphate dehydrogenase (G6PD) deficiency was performed in 5 boys and 14 girls. Out of those, $4(80 \%)$ boys and 5 (36\%) girls were found to be G6PD deficient. Our FST has a cut-off point of 2.1 Units/gram Hemoglobin, which indicates these cases are moderate to severe G6PD deficiency. All these cases were either asymptomatic or with a 
mild COVID-19. The G6PD deficiency is overrepresented in this small case series as the prevalence of G6PD deficiency in Al-Ahsa is $23 \%$ in males and $13 \%$ in females [2]. We and others anticipate that G6PD deficient individuals will be more vulnerable to severe acute respiratory syndrome coronavirus 2 , the causative agent of the COVID-19 pandemic $[3,4]$. Still, this needs to be confirmed in a large-scale population-based study.

\section{References}

[1] Saudi Press Agency. Ministry of Health reports 17 new cases of Coronavirus. 2020 (https://www.spa.gov.sa/viewfullstory.php?lang=en\&newsid=2047203).

[2] Al-Abdi SY, Alsaigh AS, Aldawoud FL, Al Sadiq AA. Lower reference limits of quantitative cord glucose-6-phosphate dehydrogenase estimated from healthy term neonates according to the clinical and laboratory standards institute guidelines: a cross sectional retrospective study. BMC pediatrics. 2013;13:137.

[3] Al-Abdi S, Al-Aamri M. G6PD deficiency in the COVID-19 pandemic: Ghost within Ghost. Hematology/Oncology and Stem Cell Therapy. 2020.

[4] Aydemir D, Ulusu NN. Is glucose-6-phosphate dehydrogenase enzyme deficiency a factor in Coronavirus-19 (COVID-19) infections and deaths? Pathogens and Global Health. 2020;114:109-10. 